\title{
Can mental health diagnoses in administrative data be used for research? A systematic review of the accuracy of routinely collected diagnoses
}

\author{
Katrina A. S. Davis ${ }^{1}$, Cathie L. M. Sudlow ${ }^{2}$ and Matthew Hotopf ${ }^{1,3^{*}}$
}

\begin{abstract}
Background: There is increasing availability of data derived from diagnoses made routinely in mental health care, and interest in using these for research. Such data will be subject to both diagnostic (clinical) error and administrative error, and so it is necessary to evaluate its accuracy against a reference-standard. Our aim was to review studies where this had been done to guide the use of other available data.

Methods: We searched PubMed and EMBASE for studies comparing routinely collected mental health diagnosis data to a reference standard. We produced diagnostic category-specific positive predictive values (PPV) and Cohen's kappa for each study.

Results: We found 39 eligible studies. Studies were heterogeneous in design, with a wide range of outcomes. Administrative error was small compared to diagnostic error. PPV was related to base rate of the respective condition, with overall median of $76 \%$. Kappa results on average showed a moderate agreement between source data and reference standard for most diagnostic categories (median kappa $=0.45-0.55$ ); anxiety disorders and schizoaffective disorder showed poorer agreement. There was no significant benefit in accuracy for diagnoses made in inpatients.

Conclusions: The current evidence partly answered our questions. There was wide variation in the quality of source data, with a risk of publication bias. For some diagnoses, especially psychotic categories, administrative data were generally predictive of true diagnosis. For others, such as anxiety disorders, the data were less satisfactory. We discuss the implications of our findings, and the need for researchers to validate routine diagnostic data.
\end{abstract}

Keywords: Psychiatry, Diagnosis, Population research, Administrative data, Electronic health records, Case registers, Hospital episode statistics

\section{Background}

Databases such as those produced by electronic health records or for reimbursement of medical costs, contain routinely collected data on diagnosis that has considerable application in research, such as for ascertaining outcomes in epidemiology or identifying suitable research participants for clinical trials [1-3]. There has been a

\footnotetext{
* Correspondence: matthew.hotopf@kcl.ac.uk

'Department of Psychological Medicine, Institute of Psychiatry Psychology and Neuroscience, Kings College London, London, UK

${ }^{3}$ Department of Psychological Medicine and SLaM/loPPN BRC, Kings College London, PO62, Weston Education Centre, Cutcombe Road, London SE5 9RJ, UK

Full list of author information is available at the end of the article
}

long history of using routine data in mental health research, from the earliest studies of asylum records through to the 'case register' of the 20th century [4]. The easy availability of large volumes of data regarding patients with mental health diagnoses from routine clinical practice following the shift to electronic health records can be utilised for research $[5,6]$, and massed electronically produced administrative data has been used by a diverse range of groups, using routinely collected diagnosis to identify cases of mental illness for public health and advocacy [7-10]. 
Biobanks may also link to administrative databases: connecting genomic, physiological and self-report data with hospital episodes and death registration, to become powerful tools to gain insight into risk and protective factors of a wide range of diseases. UK Biobank recruited 500,000 people aged between 40 and 69 years in 20062010 from across the UK [11], and data linkage includes to Hospital Episode Statistics (HES) in England, and the equivalent datasets in Scotland and Wales, which log every hospital admission, including to psychiatric hospitals, and include ICD-10 diagnosis codes (WHO's International Classification of Diseases) [12]. Such linkages provide a means of greatly enriching UK Biobank's outcomes in a cost-effective and scalable manner, and there would be the opportunity for identifying cases of psychiatric illness through ICD-10 codes from HES and other records. Similar data linkages are in place for other large studies [4].

Despite the promise of data linkage, there are inevitably concerns that routine data, collected for nonresearch purposes, may be prone to misclassification. Accuracy can be affected by errors at a number of points, broadly described as "diagnostic error" and "administrative error". Diagnostic error occurs when the clinician fails to find the signs/symptoms of the correct condition, makes a diagnosis not supported by research criteria, or records a diagnosis at odds with their real conclusion. Administrative error involves issues around turning the physician diagnosis into codes (ICD in the case of Hospital Episode Statistics), and submitting these codes attached to the correct record and identifiers. "Coding" traditionally utilised trained non-clinical administrators interpreting the treating clinician's handwritten records to derive a valid ICD code for the record [13], which is inevitably error-prone - although in the age of electronic health records, where the clinicians generally assign diagnosis codes, data entry error and miscoding still occurs $[5,14,15]$.

Recent reviews of accuracy of English HES data have mainly concentrated on administrative error [1, 16, 17], and there is a lack of specific information on diagnostic accuracy for psychiatric disorders. In mental health there may be particular issues about diagnostic error, which would be reflected in evaluations of the quality of psychiatric diagnoses in other data sources $[15,18]$. This may help when considering using HES and other such administrative databases to identify cases of mental illness. A previous attempt to collate results from a variety of psychiatric databases by Byrne et al. from Kings College London in 2005, identified that papers were mostly of poor quality, and the results were too variable to give an overall view on diagnostic validity [19].

The aim of the present systematic review was to identify and collate results regarding the accuracy of diagnosis in routinely collected data from mental health settings to guide the interpretation of the use of such data to identify cases. Specifically our objectives were: to evaluate the agreement and validity of a routinely recorded diagnosis compared with a reference diagnosis for psychiatric disorders (i) in general, (ii) for different psychiatric diagnoses, and (iii) comparing diagnoses made as inpatients with outpatients.

\section{Methods}

We used Preferred Reporting Items for Systematic Reviews and Meta-Analyses (PRISMA) guidelines to develop the design, conduct and reporting of this review. One author (KD) carried out the search and extracted data.

\section{Search strategy}

We searched Medline (PubMed) and Embase from 1980 to November 2014 for studies assessing the accuracy of routinely collected data regarding psychiatric diagnosis against a reference standard diagnosis. We used a combination of medical subject heading and text word terms for 'mental health'; 'accuracy', 'reliability' and 'validity'; 'diagnosis,' ICD' and 'DSM'; and 'medical records,' 'coding' or 'registers'. We reviewed bibliographies of included publications and used Google Scholar to identify any citing papers for additional relevant reviews or studies (see Additional files 1 and 2: Figure S1 and S2 for detail of search strategy).

\section{Eligibility criteria}

Studies were included if they were a peer-reviewed published comparison of psychiatric diagnoses in routinely recorded data against reference standard diagnoses using ICD, DSM or similar psychiatric classification systems. The studies included samples of patients recruited from population, primary or secondary care settings; however, the diagnoses under study were those derived from secondary care only - either inpatient or outpatient psychiatric services. The data that was being examined (source diagnosis) could be taken from official clinical documentation ["clinical"] or from a research or administrative database. Where a clinical source diagnosis was used, the comparison data (reference diagnosis) had to be a research diagnosis ["research"] to look at diagnostic error, but where a database source diagnosis was used, clinical documentation ["chart"] could also be used for a reference diagnosis to look at administrative error. Comparing a database source diagnosis and a research reference diagnosis gives clinical and administrative error combined. Research diagnoses could be considered reference diagnoses whether they used structured casenote review and/or research interview to reach the diagnosis, as long as they conformed to Spitzer's "Longitudinal, Expert and All Data" (LEAD) diagnostic approach [20]. 
Studies were reviewed for inclusion by KD, and where there was doubt, discussed with $\mathrm{MH}$.

We assessed each eligible paper for quality using an established checklist [21] which marks studies on aims (3 marks), method (9), results \& discussion (10). There were no suggested cut-off points with this checklist, so we defined criteria for inadequate, poor, moderate and good quality using total and category-specific scores. The studies considered inadequate were those which scored less than two in any category or less than ten overall. Studies were considered good quality if they scored at least $75 \%$ of the points from each section (see Additional file 3: Table S1 for the quality rating of individual papers).

\section{Data extraction}

We devised a form to extract information from each study which included (1) the nature of the cohort studied, including clinical setting, selection criteria, location, sample size and age range; (2) source of routine diagnostic data; (3) nature of reference diagnosis, how it was derived, and any measures of reliability for this diagnosis; (4) the diagnosis, diagnostic grouping or diagnoses under study, and the diagnostic system used (e.g. ICD/ DSM); (5) the base rate for each diagnosis studied (i.e. the prevalence in the setting the diagnosis was made according to reference diagnosis); (6) measures of concordance between diagnostic data and reference diagnosis: validity measures - sensitivity, specificity, positive- and negative-predictive values - and agreement measures percentage agreement, Cohen's kappa $(\mathrm{k})$ and area under the curve.

\section{Data analysis}

After consideration of the data available from the papers, and our aim to assess the accuracy of case finding by using routine diagnosis we chose two parameters to report: (1) Positive predictive value (PPV) provides an estimate of the probability that a given diagnosis in the source data will match the reference diagnosis acting as "gold standard"; (2) Cohen's kappa provides a measure of agreement between the source data and the reference comparison. The sensitivity and negative predictive value are useful for considering representativeness and the recruitment of controls, but they are of most use when using true population studies, where unidentified cases can be found, rather than the secondary care studies identified here.

We give diagnosis-specific results at chapter level (eg "affective disorders") and disorder level (eg "bipolar affective disorder") according to the reporting in the original papers. Some papers report at both chapter level and disorder level, in which case the results for the disorder will be a subset of the results for the chapter.
Otherwise, we treated results within the same study as independent for data analysis purposes.

Using cross-tabulations provided in the source paper, or working back from accuracy statistics, a $2 \times 2$ table was constructed of true-positives, false-positives, false-negatives, and true negatives for each diagnosis studied in each paper. From this, the PPV and percentage agreement was calculated. It was thus possible to calculate a PPV for all of the specific outcome categories, even where not originally reported, with $95 \%$ confidence intervals calculated using Wilson's method [22].

Cohen's kappa was calculated from the observed and expected agreement [23]. Two difficulties were encountered: (i) where no-one without the diagnosis in source data was studied, kappa could not be calculated; (ii) where agreement was worse than chance, a negative kappa results; since the magnitude of a negative kappa is uninformative this was regarded as zero.

We did not undertake formal meta-analysis or metaregression due to the heterogeneity between studies in their methods, participant characteristics and reporting. We used non-parametric tests - Kruskall-Wallis $\mathrm{H}$ with Bonferroni correction - to assess for independence of groups for data source, and to explore setting of diagnosis. Calculations and graphs were performed using Microsoft Excel 2013 with the Real Statistics plug-in [24].

\section{Results \\ Papers}

Figure 1 shows the PRIMSA flow chart for the review. Our literature search identified 117 potential publications. Of these 72 were excluded, and a further six were found to be of inadequate quality, leaving a total of 39 [25-63]. The excluded papers and reasons for exclusion are in Additional file 4: Table S2.

Included studies are described in Additional file 5: Table S3. The publications were predominantly Scandinavian $(n=22)$ and from the USA $(n=10)$, with the four largest studies coming from Canada. They were published between 1988 and 2014 although they reflect diagnoses made up to 20 years prior to the date of publication of the studies. Many had been published with a view to using the source data for further research.

\section{Study design}

Cohorts ranged from samples of the general population to inpatients with specified working diagnosis. The prevalence of specified diagnoses in secondary care (base rates) varied widely. The number of diagnostic categories examined in each study varied between one and eight. In all, there were 16 diagnostic categories considered. In the 39 papers studied, there were 104 diagnosis-specific results. The most common diagnosis studied was schizophrenia $(n=19)$, followed by bipolar affective disorder $(n=12)$ and 


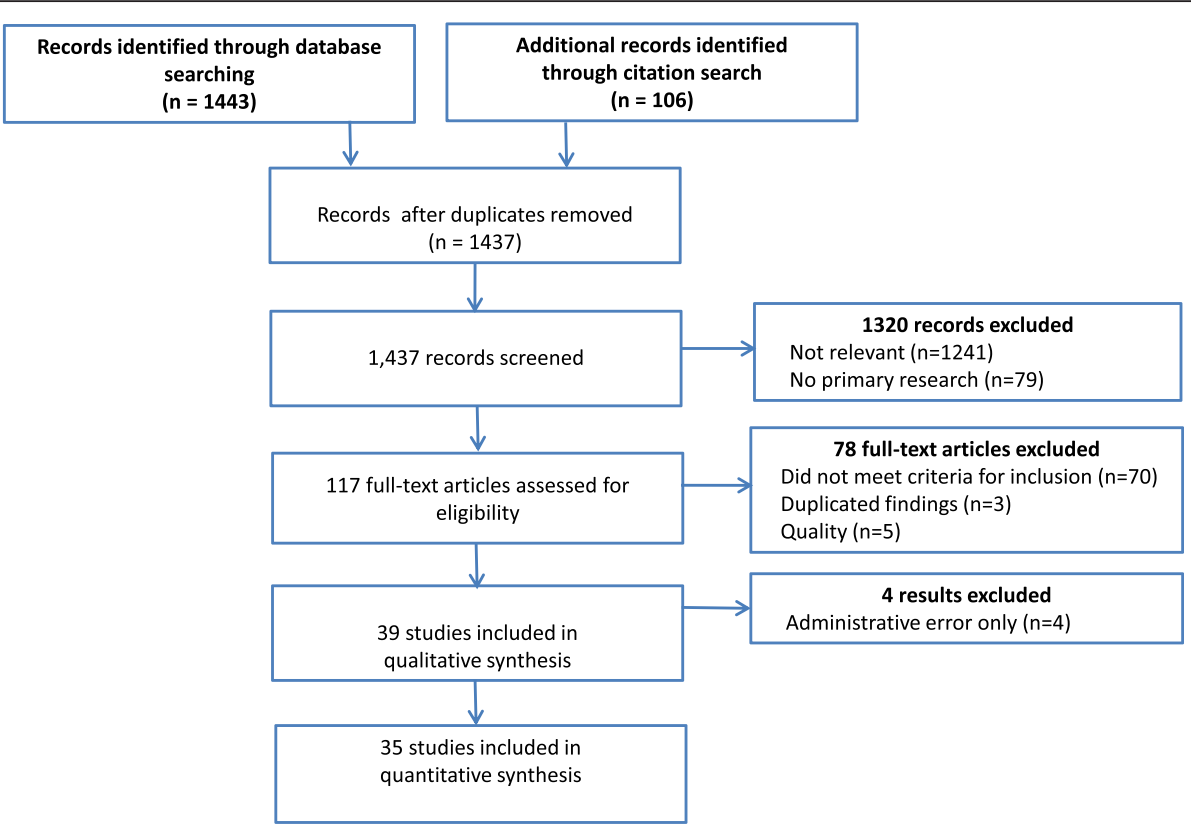

Fig. 1 Preferred Reporting Items for Systematic Reviews and Meta-Analyses (PRISMA) flow diagram of the systematic review

unipolar depression $(n=12)$. Ten results showed the overall agreement across a number of diagnoses. A number of studies used the category of "schizophrenia spectrum" $(n=13)$ to describe a group of psychotic disorders usually including schizophrenia, schizotypal disorder and schizoaffective disorder, but varying on the inclusion of other schizophreniform psychoses and delusional disorders. Since the studies were comparing like-for-like in their routine and reference diagnoses, we used the term "schizophrenia spectrum" whenever a group of nonaffective psychoses including schizophrenia was studied, without further differentiation.

The source data was derived directly from clinical notes in 13 studies (57 diagnosis-specific results), while 26 studies used databases: 17 used regional and national research databases; nine larger studies used databases created primarily for administrative purposes. The reference diagnosis was the "chart" diagnosis in four studies, and was otherwise a research diagnosis. Research diagnoses consisted of a notes review in 15, an interview in five, and an interview with notes review in 15 . Thirteen studies used more than one researcher reaching a diagnosis independently and reported the inter-rater reliability of the research diagnosis. In 11 cases, this could be compared with the kappa agreement between source and reference [33, 34, 36, 38, 44-46, 50, 57, 59, 61].

There are three groups of results: those using a database diagnosis as the source and chart diagnosis as the reference, giving administrative error only (six results from four papers); those using clinical diagnosis as the source with research diagnosis as the reference, giving diagnostic error only (57 results from 13 papers); and those using database diagnosis as the source with research diagnosis as the reference, giving administrative and diagnostic error combined (41 results from 22 papers).

Twenty-four studies examined diagnoses made as an inpatient, while 13 included diagnoses recorded as in- or out-patients; with two exclusively examining data from outpatients. Eight studies concentrated on diagnoses made at first presentation. Two studies $[40,55]$ specified that more than one entry stating the diagnosis was required for inclusion in the cohort, and a further two $[25,43]$ selected inpatients with one diagnosis, but outpatients only if they had two. Multiple instances of diagnosis were the norm in the remainder of the studies, except those of first episode, with various algorithms for treating differing diagnoses: "at least one", "last", "most often" and using a formal hierarchy. The result using the "last" diagnosis was chosen for this analysis where multiple results were given, as this was shown to be a good method [54] and thought to be most similar to where no choice in results had been given.

The source data were coded using systems from DSM versions III, III-R \& IV or ICD versions $7-10$ or local codes based on these classifications (eg a Canadian version of ICD-10 or codes specific to Veterans Affairs). Frequently the administrative diagnoses covered a long time frame, and therefore mixtures of editions were used. For example McConville collected data from 1962 to 1996, covering ICD versions 7, 8, 9 and 10 [45]. 


\section{Outcomes}

There was a wide range of PPVs, from 10 to $100 \%$ with an overall median of $76 \%$ and a negative skew. PPV is connected mathematically to the base rate of the condition, and simple linear regression confirmed a moderate positive association between PPV and prevalence $\left(r^{2}=\right.$ $0.27, p<<0.01$, correlation coefficient $(\beta)=0.40$,). Kappa was calculated for the 29 studies where a "true negative" rate was known, giving 91 diagnosis-specific results. Agreement using kappa ranged from $<0$ to 1 (i.e., from worse than chance agreement to a perfect match), and the distribution was fairly symmetrical. The median kappa was 0.49 , a value that is classed as a moderate inter-rater agreement [64]. In contrast with PPV, there was no correlation with prevalence $\left(r^{2}=0.0032, p=0.97\right)$. Due to the dependence of PPV on prevalence, kappa would be the preferred statistic when comparing between data sources with different prevalence. The kappa values can also be compared against the inter-rater reliability of the research diagnoses in eleven of the papers. In all cases the kappa result shows greater discordance for the source data than between researchers: kappa for research diagnosis was $0.71-1$, being between 1.2 to 3 times higher (median 1.7) than the results for source data. This suggests that the studies are demonstrating more than the reliability of the diagnostic codes.

The median PPV and kappa results for the administrative error group were $91 \%$ and 0.73 respectively; for the diagnostic error group $74 \%$ and 0.48 ; for the combined error group $77 \%$ and 0.36 . Kruskal Wallis pairwise testing confirmed that kappa was higher for the administrative error group versus the combined group $(p=0.006)$, and not significantly different for the diagnostic error versus the combined groups $(p=0.33)$. The significantly higher kappa agreement for the administrative error only group suggests that the error in diagnostic data overall occurs mainly at the clinical rather than the administrative stage. A few papers were able to comment directly on the relative contribution of clinical versus administrative error. Moilenan et al. [46] and Makikyro et al. [44] agreed, with clinical errors greatly outnumbering administrative ones (55 vs 9 and 16 vs 2 respectively); although Uggerby et al. [60] was at odds, with seven clinical vs 13 administrative errors in their research database.

We omitted the administrative error only group from further analysis, and the results from the diagnostic error only group and the combined error group were considered together for subsequent comparisons.

\section{Results by diagnostic group}

The Positive Predictive Value (PPV) for diagnosis for all studies is plotted by diagnostic group in the Forest Plots in Figs. 2 and 3, showing how the PPV varies by prevalence and diagnostic group amongst other variables. For those diagnostic categories with four or more results, the spread is also displayed in box plot Fig. 4a, where the range and quartile values of PPV results in the same diagnostic category can be seen. The spread of Cohen's kappa is also shown for comparison in Fig. 4b.

The highest PPV was for the broad category of "psychotic" illness. Every study agreed that in a cohort with a diagnosis of psychotic illness recorded in secondary care, at least $80 \%$ are likely to meet research criteria for this, and most suggested over $90 \%$. The diagnosis of schizophrenia shows a greater spread of PPV (40-100 \%) than psychotic illness, but the majority of studies found the diagnosis at least $75 \%$ predictive. "Schizophrenia spectrum" results lie in-between that of broad psychosis and narrow schizophrenia. Other diagnoses that have a median PPV around $75 \%$ are affective disorders (with approximately the same spread as schizophrenia), unipolar depression and bipolar affective disorder (with a wider spread). Substance misuse disorders and anxiety disorders had a lower median PPV, while the diagnosis of schizoaffective disorder had a low PPV $(<60 \%)$ in all of the five studies that included it.

The variation of kappa within diagnostic category is very large, the range being lowest for affective disorders (0.3), and highest for affective disorders and highest for schizophrenia (0.7). But between diagnostic groups the variation is small compared with PPV. The median kappa for schizophrenia and schizophrenia spectrum disorders are both around 0.5 , as are diagnoses of depression and bipolar disorder.

\section{Results by inpatient status}

We divided studies into those done exclusively on inpatient data, and those that included both inpatients and outpatients. Since around half of the studies in the inpatient group looked only at patients in their first presentation, which might be expected to have lower accuracy, we subdivided into three groups: inpatient only, first presentation only, and mixed in/outpatient. To compare them, we considered only the most common diagnostic categories: the diagnosis-specific results for schizophrenia or schizophrenia spectrum (schizophrenia used in preference where both given); unipolar depression and bipolar, or affective disorder (individual diagnoses used in preference where given); and overall agreement. There were 25 diagnoses considered in the mixed group with median PPV $72 \%$ (interquartile range 44-87), 13 results in the inpatient group with median PPV 77 \% (IQR 76-85), and 20 results from first presentation with median PPV $75 \%$ (IQR 71-93). Looking at kappa (median 0.50, 0.45 and 0.49 respectively) with Kruskall-Wallis pairwise comparison found no significant difference between inpatient and mixed, or between 1st and mixed presentations $(p>0.1)$. 


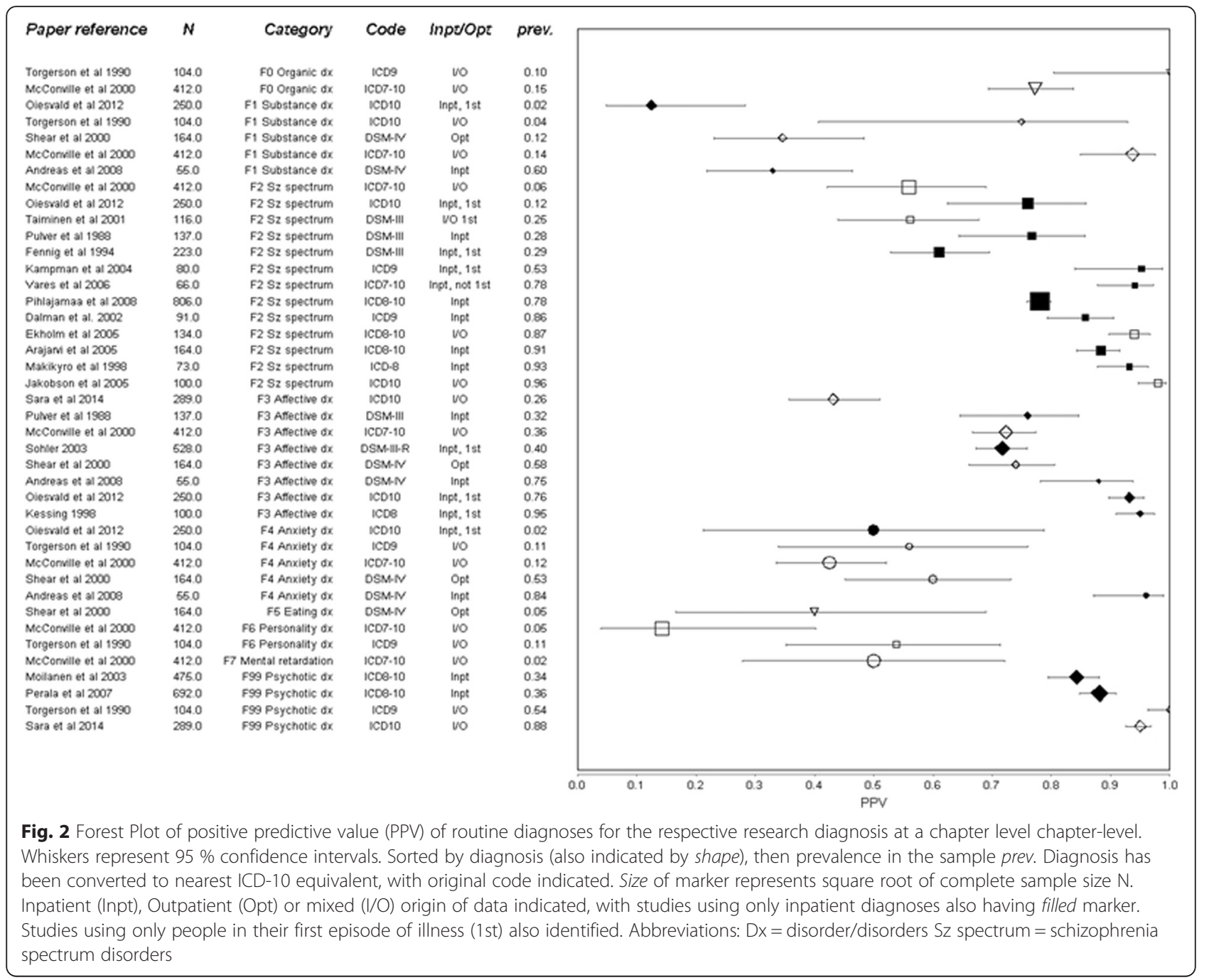

\section{Discussion}

\section{Review findings}

We found thirty-nine studies on the accuracy of routinely collected data on diagnoses in mental health, of diverse design and quality. Error appeared to be significantly greater at the clinical/diagnostic stage than in the transfer to administrative data. The spread of results for both positive predictive value and agreement (kappa), even within one diagnosis, was very large. Never the less, it could be seen that for well-defined disorders such as schizophrenia, a moderately high predictive value could be expected (median $75 \%$ ), especially when the diagnosis was made in the context of high prevalence. For diagnostic groups of anxiety and substance use disorders, and the diagnosis of schizoaffective disorder, there was on average less predictive value in the diagnosis in administrative data (although schizoaffective disorder as part of schizophrenia spectrum disorders is likely to be better). Kappa values ranged from negative to perfect, but median level for most disorders suggested moderate reliability. We did not find the expected advantage of an inpatient diagnosis, nor a disadvantage from first presentation in the most common diagnostic categories.

\section{Confidence in results}

The papers reported in this review are mainly of moderate quality. Of the recommended items in the checklist [21], the papers between them scored 64, 67 and $47 \%$ for the introduction, methods and reporting sections respectively. From a practical point of view, variations in study design hampered the integration of results and interpretation thereof. A concern for generalizability was that a sizable proportion of the papers were involved in validating a source of potential diagnoses in order to later use this source in their research, which could lead to publication bias - as there is little incentive to publish about a source that is rejected as invalid. Due to the differences in design of the larger vs smaller studies, a 


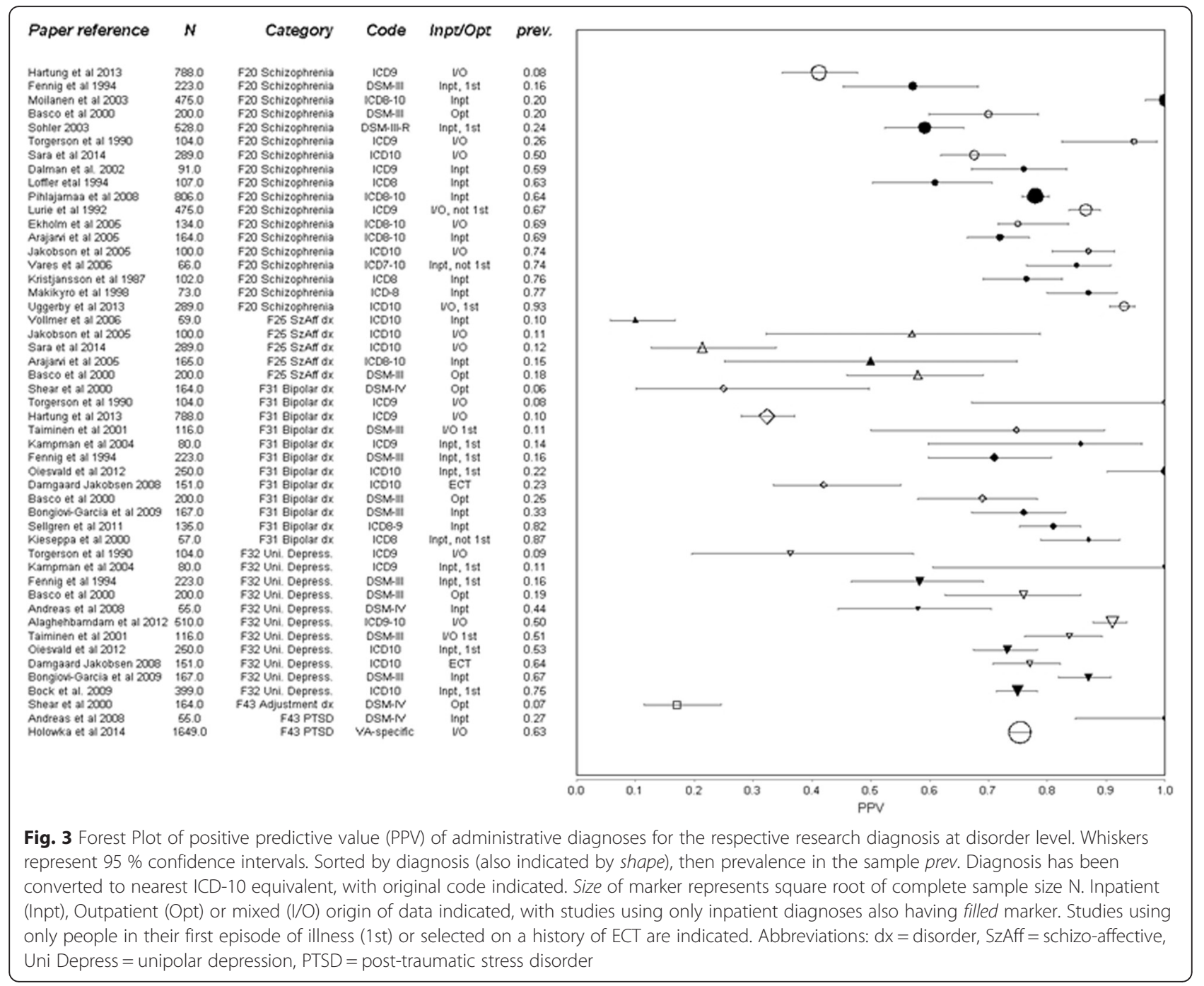

conventional analysis to look for publication bias is unlikely to be informative. The overall agreement levels of the studies compared was poorer than both predicted [65] and measured inter-rater reliability for psychiatric diagnoses, which suggests that some unfavourable results are being published.

We did not formally test for causes of heterogeneity between the studies, other than by diagnosis and inpatient status as above; but visualization of the Forest plots does not suggest a major contribution for age of study, diagnostic code used or location of study. It is possible that local clinical, administrative and other factors are major causes of heterogeneity in results that we found. Heterogeneity of study design is also likely to play a part.

A strength of our review was that we performed a comprehensive search, including forward bibliographic searching. We also decided to include a wide range of studies incorporating administrative and diagnostic error, past and present coding systems, and a large number of different psychiatric conditions. This allowed us to present the whole range of results that could be expected from a new source of diagnoses.

Weaknesses in this review are that the included studies were too heterogeneous to combine in order to adequately test for publication bias or changes over time, and the wide overlapping ranges for some of the categories that were compared leads to reduced confidence in the validity of our comparisons. We chose to concentrate on diagnoses made in secondary care, which is helpful for looking at severe mental illness, but not common disorders. We also limited our discussion to the ability to 'rule in' a disorder, rather than ruling out disorders, which is of use when creating control groups. Risk of bias was increased as the search and data extraction was carried out by just one coauthor, and we did not register the review with any database of systematic reviews. 


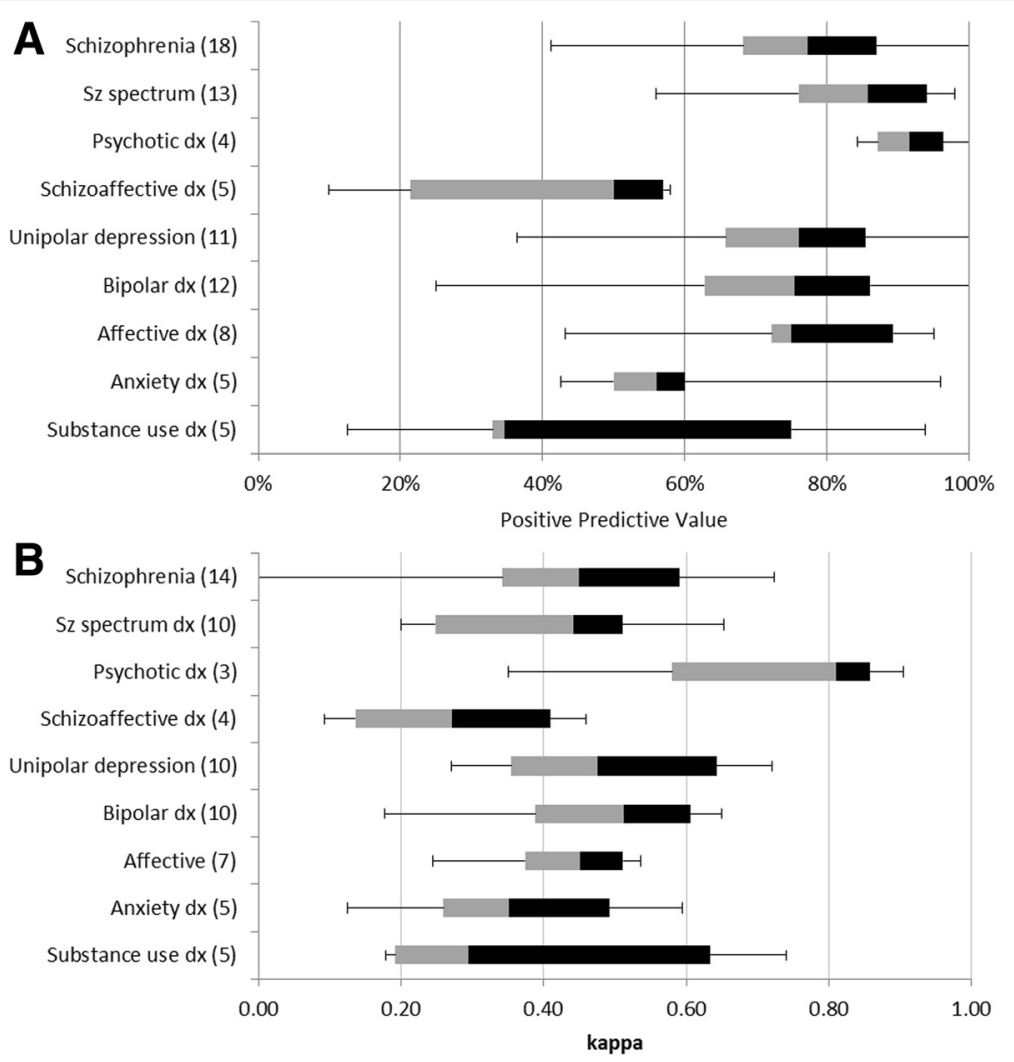

Fig. 4 Box plots summarising a positive predictive values (PPV) and $\mathbf{b}$ Cohen's kappa of diagnoses reported in four or more studies. Treating each result as a point, the median of PPVs is the transition line, the interquartile range is indicated by the box and the range of findings by the whiskers. Numbers of studies in parentheses

\section{Comparing with others}

Byrne et al. [19], who carried out the only previous review of validation studies for psychiatric diagnosis using narrower inclusion criteria, found similar disparities in the reporting of different studies, and was not able to analyse further. Some of our other findings that can be compared with previous findings include that schizophrenia and schizophrenia spectrum are relatively reliable, since Chang et al. [66] in a review of the stability of psychosis diagnoses found that people with psychosis tend to shift towards schizophrenia over time. Our finding that the major source of error between clinician and administrative database was at the clinician stage was also found in Sytema et al., a study on three registries in the 1980s [67]. 'Error' for psychiatric diagnosis in our sample was between 0 and $90 \%$, which is probably higher than would be expected for medical diagnoses. The recent review of coding in NHS hospitals (excluding psychiatry) showed error in the primary diagnosis to be between 1 and $34 \%$, with a mean of $9 \%$ [11].

\section{Psychiatric diagnosis in practice}

Assigning a psychiatric diagnosis is not straight-forward. Although training and research concentrate on discrete categories, real cases are rarely simple to describe using these categories. Making a correct diagnosis depends on the depth and duration of observation over time and the range of information available [18]. The use of structured interviews improves reliability but is not widespread $[68,69]$. There has also long been speculation that reliable categorical diagnosis in psychiatry is something of an illusion [70]. By this token, what we have termed as "diagnostic error" could be regarded as a demonstration of the weakness in the current diagnostic paradigm: the natural variation of practitioners forced to use artificial categories that do not reflect the issues of mental distress that they see.

If the "gold-standard" DSM/ICD diagnoses are not valid, then it would not be possible to calculate diagnostic validity, and our comparison would be measuring only reliability [71]. However, we have observed that inter-rater reliability for researchers is actually much higher than agreement between source and research diagnoses, meaning that error is occurring in clinical and administrative diagnosis as well as any problems there may be with the classifications per se.

While acknowledging that there is some merit in other ways of thinking about forms of normality and 
psychopathology, categorical diagnoses such as in DSM/ ICD are still used to meaningfully communicate to other clinicians, allied health professionals and GPs, and have to be acceptable to all involved $[15,65]$. Mental health diagnoses also frequently have legal consequences for individuals [72]. On a wider level, categorical diagnosis is used to inform managers and commissioners of the overall composition of a caseload. In research also, it is helpful that patients with specific sets of problems can be identified, for epidemiology, outcome studies, and recruitment into clinical trials. With the difficulties in assigning a specific diagnosis, and the diagnosis having to perform various legal, pecuniary and practical tasks, there have been reports of bias [73-76], and it should not be surprising that there is less reliability in routinely recorded diagnoses than those given by a disinterested party for research purposes.

\section{Conclusions}

Our results suggest that administrative data is variable in its accuracy for diagnosis, and it may not be possible to generalise from one data source to another. Each data source may need to be validated individually, and this will enable the researcher to choose the outcomes most pertinent for their research needs. Following specific guidelines for validation studies would assist others to benefit [21]. The biggest source of error seems to be in the reliability of the clinical diagnostic process. One can be more confident in the diagnosis of psychotic disorders in general, and schizophrenia in particular, while great caution would have to be used in our view before concluding anything from administrative data about anxiety or substance disorders, or schizoaffective disorder. There may be issues about the meaningfulness of diagnostic classification in psychiatry that deserve further research, especially around the borders of these categories.

A register of patients is easier than ever to produce. Our finding, if replicated by others, that a register/administrative diagnosis may not be significantly less accurate than one recorded routinely in clinical notes (when compared to a reference diagnosis) may be of significance to researchers who can now access structured information in anonymised and pseudo-anonymised records through research databases [5, 77].

Despite our support for using administrative data in general, it should never be used unsupported to estimate incidence, prevalence, or disease burden in a population, as they are biased towards those who recognise a problem, seek help or become unmanageable in the community. The World Health Organization estimates that $35-50 \%$ of people with mental disorders of high severity and disability have not seen a professional in the previous year [78]. Rather, we have identified that routine data from secondary care often has the power to identify likely cases of severe mental illness for further analysis. Those wishing to use administrative data for research purposes would do well to look for validity studies for their source data and their items of interest. Where there are gaps in the current evidence for commonly used sources, validation of diagnostic data should be attempted where possible.

\section{Additional files}

Additional file 1: Figure S1. Search strategy (medline). (TIF $146 \mathrm{~kb}$ )

Additional file 2: Figure S2. Search strategy (EMBASE). (TIF $570 \mathrm{~kb}$ )

Additional file 3: Table S1. Quality assessment of papers meeting inclusion criteria, after Benchimol et al. [21]. (DOCX 58 kb)

Additional file 4: Table S2. Excluded studies. (DOCX $75 \mathrm{~kb}$ )

Additional file 5: Table S3. Included studies. (DOCX $59 \mathrm{~kb}$ )

\section{Abbreviations}

DSM, diagnostic and statistical manual of mental disorders; HES, hospital episode statistics (England); ICD, WHO International Classification of Diseases; NPV, negative predictive value - probability condition absent given identified as negative; PPV, positive predictive value - probability condition present given identified as positive; PRISMA, preferred reporting items for systematic reviews and meta-analyses; $r^{2}$, residual sum of squares, also referred to as coefficient of determination

\section{Acknowledgements}

This work was supported by the the National Institute for Health Research (NIHR) Biomedical Research Centre at South London and Maudsley NHS Foundation Trust and King's College London. The views expressed are those of the author(s) and not necessarily those of the NHS, the NIHR or the Department of Health.

\section{Funding}

This work was funded by a grant from UK Biobank.

Cathie Sudlow is supported by UK Biobank and the Scottish Funding Council. Matthew Hotopf is part funded by the the National Institute for Health Research (NIHR) Biomedical Research Centre at South London and Maudsley NHS Foundation Trust and King's College London. The views expressed are those of the author(s) and not necessarily those of the NHS, the NIHR or the Department of Health.

Availability of data and materials

The datasets supporting the conclusions of this article are included within the article and its additional files.

\section{Authors' contributions}

$\mathrm{MH}$ and $\mathrm{CS}$ conceived this study. $\mathrm{MH}$ and $\mathrm{KD}$ designed this study. $\mathrm{KD}$ collated and analysed the data, with contributions from $\mathrm{MH}$. All authors were involved in the writing of the manuscript. All authors have read and approve of the final version of the manuscript.

\section{Authors' information}

The authors are members of the UK Biobank Mental Health Consortium and the MQ Data Science Group.

\section{Competing interests}

The authors declare that they have no competing interests.

Consent for publication

Not applicable.

Ethics approval and consent to participate Not applicable. 


\section{Author details}

'Department of Psychological Medicine, Institute of Psychiatry Psychology and Neuroscience, Kings College London, London, UK. ${ }^{2}$ South London and Maudsley NHS Foundation Trust, Maudsley Hospital, Denmark Hill, London SE5 8AZ, UK. ${ }^{3}$ Department of Psychological Medicine and SLaM/loPPN BRC, Kings College London, PO62, Weston Education Centre, Cutcombe Road, London SE5 9RJ, UK

\section{Received: 8 February 2016 Accepted: 5 July 2016}

Published online: 26 July 2016

\section{References}

1. Sinha S, Peach G, Poloniecki JD, Thompson MM, Holt PJ. Studies using English administrative data (Hospital Episode Statistics) to assess health-care outcomes_-systematic review and recommendations for reporting. Eur J Public Health. 2013:23(1):86-92.

2. Perlis RH, losifescu DV, Castro VM, Murphy SN, Gainer VS, Minnier J, Cai T, Goryachev S, Zeng Q, Gallagher PJ, et al. Using electronic medical records to enable large-scale studies in psychiatry: treatment resistant depression as a model. Psychol Med. 2012:42(01):41-50.

3. Alaghehbandan R, MacDonald D. Use of administrative health databases and case definitions in surveillance of depressive disorders. a review. OA Epidemiology 2013;1(1):3

4. Munk-Jørgensen P, Okkels N, Golberg D, Ruggeri M, Thornicroft G. Fifty years' development and future perspectives of psychiatric register research. Acta Psychiatr Scand. 2014;130(2):87-98.

5. Castillo EG, Olfson M, Pincus HA, Vawdrey D, Stroup TS. Electronic Health Records in Mental Health Research: A Framework for Developing Valid Research Methods. Psychiatr Serv. 2015;66(2):193-6.

6. Stewart R. The big case register. Acta Psychiatr Scand. 2014;130(2):83-6.

7. RSA Open Public Services Network. In: RSA OPSN Mental Health, Mind, editor. Exploring how available NHS data can be used to show the inequality gap in mental healthcare. 2015.

8. The Information Centre for Health and Social Care, (now NHS Digital). In: NHS, editor. Users and Uses of Hospital Episode Statistics. 2012

9. Tricco AC, Pham B, Rawson NS. Manitoba and Saskatchewan administrative health care utilization databases are used differently to answer epidemiologic research questions. J Clin Epidemiol. 2008;61(2):192-7.

10. Spiranovic C, Matthews A, Scanlan J, Kirkby KC. Increasing knowledge of mental illness through secondary research of electronic health records: opportunities and challenges. Adv Ment Health. 2016;14(1):14-25.

11. Sudlow C, Gallacher J, Allen N, Beral V, Burton P, Danesh J, Downey P, Elliott P, Green J, Landray M. UK Biobank: an Open Access resource for identifying the causes of a wide range of complex diseases of middle and old age. PLoS Med. 2015;12(3):1-10.

12. Adamska L, Allen N, Flaig $R$, Sudlow $C$, Lay M, Landray M. Challenges of linking to routine healthcare records in UK Biobank. Trials. 2015;16 Suppl 2:068.

13. Dixon J, Sanderson C, Elliott P, Walls $P$, Jones J, Petticrew M. Assessment of the reproducibility of clinical coding in routinely collected hospital activitydata: a study in two hospitals. J Public Health. 1998;20(1):63-9.

14. Jasser SA, Garvin JH, Wiedemer N, Roche D, Gallagher RM. Information Technology in Mental Health Research: Impediments and Implications in One Chronic Pain Study Population. Pain Med. 2007:8:S176-81.

15. Whooley O. Diagnostic ambivalence: psychiatric workarounds and the Diagnostic and Statistical Manual of Mental Disorders. Sociol Health IIIn. 2010;32(3):452-69.

16. Burns EM, Rigby E, Mamidanna R, Bottle A, Aylin P, Ziprin P, Faiz OD Systematic review of discharge coding accuracy. J Public Health. 2012;34(1):138-48

17. CAPITA. The quality of clinical coding in the NHS. In: Payment by Results data assurance framework 2014.

18. Aboraya A, First MB. The Reliability of Psychiatric Diagnoses: Point-Our psychiatric diagnoses are still unreliable = Counterpoint-There isn't enough evidence in clinical settings. Psychiatry (Edgmont (Pa:Township)). 2007:4(1):22-5

19. Byrne N, Regan C, Howard L. Administrative registers in psychiatric research: a systematic review of validity studies. Acta Psychiatr Scand. 2005;112(6):409-14.

20. Spitzer RL. Psychiatric diagnosis: Are clinicians still necessary? Compr Psychiatry. 1983;24(5):399-411.
21. Benchimol El, Manuel DG, To T, Griffiths AM, Rabeneck L, Guttmann A Development and use of reporting guidelines for assessing the quality of validation studies of health administrative data. J Clin Epidemiol. 2011;64(8):821-9.

22. Wallis S. Binomial Confidence Intervals and Contingency Tests: Mathematical Fundamentals and the Evaluation of Alternative Methods. J Quant Linguist. 2013;20(3):178-208.

23. Cohen J. A Coefficient of Agreement for Nominal Scales. Educ Psychol Meas. 1960;20(1):37-46.

24. Zaiontz C. Real Statisitcs Resource Pack 3.5 www.real-statistics.com. Accessed 23 Oct 2015. In. Release 3.5 edn; 2013-2015.

25. Alaghehbandan R, MacDonald D, Barrett B, Collins K, Chen Y. Using Administrative Databases in the Surveillance of Depressive Disorders-Case Definitions. Popul Health Manag. 2012;15(6):372-80.

26. Andreas $S$, Theisen $P$, Mestel $R$, Koch $U$, Schulz $H$. Validity of routine clinical DSM-IV diagnoses (Axis I/II) in inpatients with mental disorders. Psychiatry Res. 2009:170(2-3):252-5.

27. Arajärvi R, Suvisaari J, Suokas J, Schreck M, Haukka J, Hintikka J, Partonen T, Lönnqvist J. Prevalence and diagnosis of schizophrenia based on register, case record and interview data in an isolated Finnish birth cohort born 1940-1969. Soc Psychiatry Psychiatr Epidemiol. 2005:40(10):808-16.

28. Basco MR, Bostic JQ, Davies D, Rush AJ, Witte B, Hendrickse W, Barnett V. Methods to improve diagnostic accuracy in a community mental health setting. Am J Psychiatr. 2000;157(10):1599-605.

29. Bock C, Bukh J, Vinberg M, Gether U, Kessing L. Validity of the diagnosis of a single depressive episode in a case register. Clin Pract Epidemiol Ment Health. 2009;5(1):4.

30. Bongiovi-Garcia ME, Merville J, Almeida MG, Burke A, Ellis S, Stanley BH, Posner K, Mann JJ, Oquendo MA. Comparison of clinical and research assessments of diagnosis, suicide attempt history and suicidal ideation in major depression. J Affect Disord. 2009;115(1-2):183-8.

31. Dalman C, Broms J, Cullberg J, Allebeck P. Young cases of schizophrenia identified in a national inpatient register. Soc Psychiatry Psychiatr Epidemiol. 2002;37(11):527-31.

32. Damgaard Jakobsen K, Hansen T, Dam H, Bundgaard Larsen E, Gether U, Werge T. Reliability of clinical ICD-10 diagnoses among electroconvulsive therapy patients with chronic affective disorders. Eur J Psychiatry. 2008;22:161-72.

33. Ekholm B, Ekholm A, Adolfsson R, Vares M, Ösby U, Sedvall GC, Jönsson EG. Evaluation of diagnostic procedures in Swedish patients with schizophrenia and related psychoses. Nord J Psychiatry. 2005;59(6):457-64.

34. Fennig S, Craig TJ, Tanenberg-Karant M, Bromet EJ. Comparison of facility and research diagnoses in first-admission psychotic patients. Am J Psychiatry. 1994;151(10):1423-9.

35. Hartung DM, Middleton L, McFarland BH, Haxby DG, McDonagh MS, McConnell J. Use of administrative data to identify off-label use of second-generation antipsychotics in a medicaid population. Psychiatr Serv. 2013:64(12):1236-42

36. Holowka DW, Marx BP, Gates MA, Litman HJ, Ranganathan G, Rosen RC, Keane TM. PTSD diagnostic validity in Veterans Affairs electronic records of Iraq and Afghanistan veterans. J Consult Clin Psychol. 2014:82(4):569-79.

37. Jakobsen KD, Frederiksen JN, Hansen T, Jansson LB, Parnas J, Werge T. Reliability of clinical ICD-10 schizophrenia diagnoses. Nord J Psychiatry. 2005;59(3):209-12

38. Kampman $\mathrm{O}$, Kiviniemi $\mathrm{P}$, Koivisto $\mathrm{E}$, Väänänen J, Kilkku $\mathrm{N}$, Leinonen $\mathrm{E}$ Lehtinen K. Patient characteristics and diagnostic discrepancy in first-episode psychosis. Compr Psychiatry. 2004;45(3):213-8.

39. Kessing LV. Validity of diagnoses and other clinical register data in patients with affective disorder. Eur Psychiatry. 1998;13(8):392-8.

40. Kieseppa T, Partonen T, Kaprio J, Lonnqvist J. Accuracy of register- and record-based bipolar I disorder diagnoses in Finland; a study of twins. Acta Neuropsychiatr. 2000;12(3):106-9.

41. Kristjansson E, Allebeck P, Wistedt B. Validity of the diagnosis schizophrenia in a psychiatric inpatient register: A retrospective application of DSM-III criteria on ICD-8 diagnoses in Stockholm county. Nord J Psychiatry. 1987; 41(3):229-34.

42. Löffler W, Häfner H, Fätkenheuer B, Maurer K, Riecher-Rössler A, Lützhøft J Skadhede S, Munk-Jørgensen P, Strömgren E. Validation of Danish case register diagnosis for schizophrenia. Acta Psychiatr Scand. 1994;90(3):196-203.

43. Lurie N, Popkin M, Dysken M, Moscovice I, Finch M. Accuracy of Diagnoses of Schizophrenia in Medicaid Claims. Psychiatr Serv. 1992;43(1):69-71. 
44. Mäkikyrö T, Isohanni M, Moring J, Hakko H, Hovatta I, Lönnqvist J. Accuracy of register-based schizophrenia diagnoses in a genetic study. Eur Psychiatry. 1998;13(2):57-62.

45. McConville $P$, Walker NP. The reliability of case register diagnoses: a birth cohort analysis. Soc Psychiatry Psychiatr Epidemiol. 2000;35(3):121-7.

46. Moilanen K, Veijola J, Läksy K, Mäkikyrö T, Miettunen J, Kantojärvi L, Kokkonen P, Karvonen JT, Herva A, Joukamaa M, et al. Reasons for the diagnostic discordance between clinicians and researchers in schizophrenia in the Northern Finland 1966 Birth Cohort. Soc Psychiatry Psychiatr Epidemiol. 2003:38(6):305-10.

47. Oiesvold T, Nivison M, Hansen V, Sorgaard K, Ostensen L, Skre I. Classification of bipolar disorder in psychiatric hospital. A prospective cohort study. BMC Psychiatry. 2012;12(1):13.

48. Perälä J, Suvisaari J, Saarni SI, et al. LIfetime prevalence of psychotic and bipolar i disorders in a general population. Arch Gen Psychiatry. 2007;64(1):19-28.

49. Pihlajamaa J, Suvisaari J, Henriksson M, Heilä H, Karjalainen E, Koskela J, Cannon M, Lönnqvist J. The validity of schizophrenia diagnosis in the Finnish Hospital Discharge Register: Findings from a 10-year birth cohort sample. Nord J Psychiatry. 2008;62(3):198-203.

50. Pulver AE, Carpenter WT, Adler L, McGrath J. Accuracy of the diagnoses of affective disorders and schizophrenia in public hospitals. Am J Psychiatr. 1988;145(2):218-20.

51. Quan H, Li B, Duncan Saunders L, Parsons GA, Nilsson Cl, Alibhai A, Ghali WA, IMECCHI Investigators. Assessing Validity of ICD-9-CM and ICD-10 Administrative Data in Recording Clinical Conditions in a Unique Dually Coded Database. Health Serv Res. 2008:43(4):1424-41.

52. Rawson NS, Malcolm E, D'Arcy C. Reliability of the recording of schizophrenia and depressive disorder in the Saskatchewan health care datafiles. Soc Psychiatry Psychiatr Epidemiol. 1997;32(4):191-9.

53. Robinson JR, Tataryn D. Reliability of the Manitoba Mental Health Management Information System for Research. Can J Psychiatry. 1997:42(7):744-9.

54. Sara G, Luo L, Carr V, Raudino A, Green M, Laurens K, Dean K, Cohen M, Burgess $P$, Morgan V. Comparing algorithms for deriving psychosis diagnoses from longitudinal administrative clinical records. Soc Psychiatry Psychiatr Epidemiol. 2014;49:1729-37.

55. Sellgren C, Landén M, Lichtenstein P, Hultman CM, Långström N. Validity of bipolar disorder hospital discharge diagnoses: file review and multiple register linkage in Sweden. Acta Psychiatr Scand. 2011;124(6):447-53.

56. Shear MK, Greeno C, Kang J, Ludewig D, Frank E, Swartz HA, Hanekamp M. Diagnosis of nonpsychotic patients in community clinics. Am J Psychiatr. 2000;157(4):581-7.

57. Sohler NP, Bromet EP. Does racial bias influence psychiatric diagnoses assigned at first hospitalization? Soc Psychiatry Psychiatr Epidemiol. 2003;38(8):463-72.

58. Taiminen T, Ranta K, Karlsson H, Lauerma H, Leinonen K-M, Wallenius E, Kaljonen A, Salokangas RKR. Comparison of clinical and best-estimate research DSM-IV diagnoses in a Finnish sample of first-admission psychosis and severe affective disorder. Nord J Psychiatry. 2001;55(2):107-11.

59. Torgersen T, Rosseland LA, Malt UF. Coding guidelines for ICD-9 section on mental disorders and reliability of chart clinical diagnoses. Acta Psychiat Scand. 1990;81(1):62-7.

60. Uggerby P, Østergaard SD, Røge R, Correll CU, Nielsen J. The validity of the schizophrenia diagnosis in the Danish Psychiatric Central Research Register is good. Dan Med J. 2013;60(2):A4578.

61. Vares M, Ekholm A, Sedvall GC, Hall H, Jönsson EG. Characterization of Patients with Schizophrenia and Related Psychoses: Evaluation of Different Diagnostic Procedures. Psychopathology. 2006;39(6):286-95.

62. Vollmer-Larsen A, Jacobsen TB, Hemmingsen R, Parnas J. Schizoaffective disorder- the reliability of its clinical diagnostic use. Acta Psychiatr Scand. 2006;113(5):402-7.

63. Walkup J, Boyer C, Kellermann S. Reliability of Medicaid Claims Files for Use in Psychiatric Diagnoses and Service Delivery. Adm Policy Ment Health. 2000;27(3):129-39

64. Landis JR, Koch GG. The measurement of observer agreement for categorical data. Biometrics. 1977;33(1):159-74.

65. Sartorius N, Ustun TB, Korten A, Cooper JE, van Drimmelen J. Progress toward achieving a common language in psychiatry, II: Results from the international field trials of the ICD-10 diagnostic criteria for research for mental and behavioral disorders. Am J Psychiatry. 1995;152(10):1427-37.

66. Chang WC, Chan SSM, Chung DWS. Diagnostic stability of functional psychosis: A systematic review. Hong Kong J Psychiatry. 2009;19(1):30-41.
67. Sytema S, Giel R, Horn GHMMT, Balestrieri M, Davies N. The reliability of diagnostic coding in psychiatric case registers. Psychol Med. 1989;19(04):999-1006.

68. Brugha TS, Bebbington PE, Jenkins R. A difference that matters: comparisons of structured and semi-structured psychiatric diagnostic interviews in the general population. Psychol Med. 1999;29(5):1013-20.

69. Miller PR, Dasher R, Collins R, Griffiths P, Brown F. Inpatient diagnostic assessments: 1. Accuracy of structured vs. unstructured interviews. Psychiatry Res. 2001;105(3):255-64.

70. Double D. The limits of psychiatry. Br Med J. 2002;324(7342):900-4.

71. Zachar P, Jablensky A. Introduction: The concept of validation in psychiatry and psychology. In: Alternative Perspectives on Psychiatric Validation: DSM, ICD, RDoC, and Beyond. edn. Edited by Peter Zachar, Drozdstoj St. Stoyanov, Massimiliano Aragona, Jablensky A. Oxford: Oxford University Press; 2015:3-46.

72. Noah L. Pigeonholing IIIness: Medical Diagnosis as a Legal Construct. Hastings Law J. 1999;50:241.

73. Grann M, Holmberg G. Follow-Up of Forensic Psychiatric Legislation and Clinical Practice in Sweden 1988 to 1995. Int J Law Psychiatry. 1999:22(2):125-31.

74. Gurland BJ, Fleiss JL, Sharpe L, Roberts P, Cooper JE, Kendell RE. Cross-national study of diagnosis of mental disorders: Hospital diagnoses and hospital patients in New York and London. Compr Psychiatry. 1970;11(1):18-25.

75. Kiejna A, Misiak B, Zagdanska M, Drapala J, Piotrowski P, Szczesniak D, Chladzinska-Kiejna S, Cialkowska-Kuzminska M, Frydecka D. Money matters: does the reimbursement policy for second-generation antipsychotics influence the number of recorded schizophrenia patients and the burden of stigmatization? Soc Psychiatry Psychiatr Epidemiol. 2014;49(4):531-9.

76. Rost K, Smith R, Matthews DB, Guise B. The deliberate misdiagnosis of major depression in primary care. Arch Fam Med. 1994;3(4):333-7.

77. Stewart R, Soremekun M, Perera G, Broadbent M, Callard F, Denis M Hotopf M, Thornicroft G, Lovestone S. The South London and Maudsley NHS Foundation Trust Biomedical Research Centre (SLAM BRC) case register: development and descriptive data. BMC Psychiatry. 2009;9(1):51.

78. World Mental Health Survey Consortium WHO. Prevalence, severity, and unmet need for treatment of mental disorders in the world health organization world mental health surveys. JAMA. 2004:291(21):2581-90.

\section{Submit your next manuscript to BioMed Central and we will help you at every step:}

- We accept pre-submission inquiries

- Our selector tool helps you to find the most relevant journal

- We provide round the clock customer support

- Convenient online submission

- Thorough peer review

- Inclusion in PubMed and all major indexing services

- Maximum visibility for your research

Submit your manuscript at www.biomedcentral.com/submit
Biomed Central 